A C T A C H E I CA SCA N D I A VICA 12 (1958) $615-623$

\title{
Separation of Acetylated Sugars and Related Compounds by Paper Chromatography in Organic Two-phase Solvent Systems
}

\author{
B ÖR J E W I C K B ER G
}

\author{
Organisk-kemiska Institutionen, Kungl. Tekniska Högskolan, Stockholm, Sweden
}

\begin{abstract}
The paper chromatography of acetates of sugars, sugar alcohols, cyclitols and glycosides using two-phase organic solvent systems has been investigated. Dimethyl sulphoxide, formamide and dimethyl formamide have been used as polar solvents but though all of them gave about the same resolution, dimethyl sulphoxide was preferred for practical reasons. The mobilities were found to be very dependent on the structure of the compounds but were little influenced by the molecular weight. Exceptionally great differences in mobilities were observed with the sugar alcohol derivatives. Of the fully acetylated sugars and glycosides of the D-series which were investigated, all $\alpha$-anomers travelled faster than the corresponding $\beta$-anomers. The 2,3,6-tri- $O$-methyl derivatives of $D$-glucose and $D$-mannose which are difficult to distinguish by ordinary paper chromatography and by electrophoresis were easily separated as their acetates. This method should be of general value for the separation and characterisation of $O$-mothyl sugars.
\end{abstract}

Tn the paper chromatography of carbohydrates the relative $R_{F}$ values in Ithe aqueous solvent systems commonly used generally vary little with the solvent composition. This is a serious limitation of the method as there is usually little hope of radically improving a poor resolution by changing solvents and further, the characterisation of carbohydrate compounds by paper chromatography only may be misleading. Sometimes these difficulties are overcome in indirect ways as is the case with the sugar aleohols. These may be converted into anhydrides which are then characterised by $R_{F}$ values and colour reactions ${ }^{1}$ but, the method is time-consuming and leaves the problem of isolating the original alcohols unsolved.

In connection with work on the carbohydrate constituents of some lichens and algae which contain complicated mixtures of sugar alcohols, cyclitols and glycosides, some attempts were made to find a complementary method for the paper chromatographic separation and characterisation of such compounds.

As further search for suitable aqueous mixtures seemed unprofitable recourse was made to two-phase organic solvent systems, such as have been 
Table 1. Relative mobilities $\left(R_{\mathrm{GA}}\right)^{\mathrm{a}}$ of some sugars and sugar alcohol acetates in various solvents.

\begin{tabular}{|c|c|c|c|c|c|c|c|c|c|c|}
\hline \multirow[t]{2}{*}{ Solvents: } & \multicolumn{3}{|c|}{$\begin{array}{l}\text { Light } \\
\text { petroleum }\end{array}$} & \multicolumn{2}{|c|}{$\begin{array}{l}\text { Isopropyl } \\
\text { ether }\end{array}$} & & \multicolumn{2}{|c|}{$\begin{array}{l}\text { Ethyl } \\
\text { ether }\end{array}$} & \multicolumn{2}{|c|}{ Benzene } \\
\hline & \multicolumn{2}{|c|}{ FA DMF() } & DMS & $\mathbf{F A}$ & DMFed) & DMS & \multicolumn{2}{|c|}{ FA DMS } & FA & DMS \\
\hline Compound b: & 2.0 & 3.0 & 1.9 & 1.2 & 1.3 & 1.2 & 1.1 & 1.1 & 1.0 & 1.0 \\
\hline $\begin{array}{l}\text { D-D-Xylose } \\
1,2,3,4,5,6-O \cdot A c \\
D-\text { sorbitol }\end{array}$ & 1.1 & 0.8 & 1.2 & 1.1 & 0.9 & 1.0 & 1.0 & 1.0 & 1.1 & 1.0 \\
\hline $\begin{array}{l}1,2,3,4,5,6-O-A c \\
\text { D-mannitol }\end{array}$ & 3,8 & 2.5 & 4.0 & 1.6 & 1.3 & 1.4 & 1.2 & 1.2 & 1.1 & 1.0 \\
\hline $\begin{array}{l}1,2,3,6, \\
2^{\prime}, 3^{\prime}, 4^{\prime}, 6^{\prime} \cdot O-A c \\
\beta \text {-maltose }\end{array}$ & 0.1 & - & 0.1 & 0.4 & - & 0.6 & 0.9 & 0.8 & 1.1 & 1.0 \\
\hline 1,2,3,4-O-Ac & 0.0 & - & 0.0 & 0.1 & - & 0.1 & 0.3 & 0.4 & 0.1 & 0.9 \\
\hline $\begin{array}{l}2,3,4,6-O-A c \\
\alpha, \beta-\mathrm{D}-\text { glucose }\end{array}$ & 0.0 & - & 0.0 & $\begin{array}{l}0.4 \\
0.1\end{array}$ & - & $\begin{array}{l}0.2 \\
0.1 \\
\end{array}$ & $\begin{array}{l}0.5 \\
0.2\end{array}$ & $\begin{array}{l}0.5 \\
0.3\end{array}$ & 0.1 & 0.9 \\
\hline $\begin{array}{l}R_{F-\text { values of }} \\
1,2,3,4,6-O \text {-Ac } \\
a-\mathrm{D} \text {-glucose }\end{array}$ & 0.05 & 0.01 & 0.07 & 0.4 & 0.4 & 0.45 & 0.7 & 0.6 & 0.85 & 0.6 \\
\hline \multicolumn{6}{|c|}{ a $R_{\mathrm{GA}}=\frac{\text { Movement of compound }}{\text { Movement of } 1,2,3,4,6 \text {-penta- } O \text {-acetyl } \alpha \text {-D-glucose }}$} & & & & & \\
\hline \multicolumn{11}{|c|}{$\begin{array}{l}\text { b } 1,2,3,4-O-A c a-D-x y l o s e \text { stands for } 1,2,3,4 \text {-tetra- } O \text {-acetyl } \alpha \text {-D-xylose etc. } \\
\text { c Elongated spots }\end{array}$} \\
\hline
\end{tabular}

successfully employed for the separation of various lipophilic compounds, e.g..$^{2-6}$. In addition to formamide (FA) and dimethyl formamide (DMF) which have been used earlier, dimethyl sulphoxide (DMS) also was tried as the polar stationary phase. Attempts were first made to separate some hexoses using $25 \% \mathrm{v} / \mathrm{v}$ solutions of FA or DMS in ethyl acetate as solvents but as the relative $R_{F}$ values were about the same as in the usual aqueous solvents and the spots produced in the case of DMS rather trailing, the experiments were continued with acetylated sugars.

A method for the separation of sugar acetates by reversed phase chromatography on acetylated paper has been described by Micheel and Schweppe ? but though monosaccharide acetates were well separated from disaccharide acetates the resolution within each of these groups was generally rather poor.

Preliminary experiments were made using FA, DMF and DMS as stationary solvents and light petroleum, isopropyl ether, ethyl ether and benzene as mobile solvents (Table 1). No precautions were taken against atmospheric moisture in the chromatogram tanks. 
It is evident that despite significant differences in some cases the stationary phases give on the whole about the same resolution. FA has the advantage of being sparingly soluble in all the mobile solvents and is thus strongly retained by the paper but on the other hand the silver nitrate spray (see Experimental part) cannot be used in this case for detection of the spots. DMF and DMS do not interfere with any of the spray reagents but they are less firmly held by the paper. DMS gives distinct spots in combination with light petroleum, isopropyl ether and slightly moist ethyl ether but elongated spots and very diffuse solvent fronts with sodium-dried ethyl ether and with benzene. DMF gave trailing spots with both light petroleum and isopropyl ether and was partly washed off the paper by the latter solvent.

As spraying with silver nitrate - sodium hydroxide is the most convenient way of detecting the spots, DMS is the most satisfactory of the stationary solvents tried. It is cheap and sufficiently stable when stored in glass bottles and as far as is known to the author toxic effects have not been observed. However, it should be handled with some care as when inhaled or absorbed through the skin it may produce a somewhat annoying sensation of taste.

In the following the discussion of the chromatographic technique is based mainly upon experience from work with DMS, but the same conclusions will most probably be valid for other solvents of the same type.

Before use it was necessary to impregnate the papers with the stationary solvent by dipping in a solution of the latter, blotting and drying. In order to minimise the effect of accidental irregularities in the process two such treatments with a dilute solution were preferred to one treatment with a more concentrated.

It was found that a suitable loading with DMS was obtained by using a ca $25 \% \mathrm{v} / \mathrm{v}$ solution in toluene. If the concentration was below $20 \%$ the spots became elongated or trailed and if it was above $30 \%$ the paper had a wet and spotted appearance and the solvent front tended to be uneven. When drying the papers after impregnation DMS tended to evaporate faster along the edges, causing a faster flow through these parts during development. The drying time should therefore not be prolonged unduly.

In view of the hygroscopicity of the stationary solvents it is necessary to take precautions against atmospheric moisture when handling the impregnated chromatograms as otherwise the $R_{F}$ values and resolution will vary with the weather. As is to be expected the $R_{F}$ values increase with the moisture content of the solvents and with the polarity of the mobile phase. With rising $R_{F}$ values the resolution generally decreases and maximum selectivity was therefore obtained by running the chromatograms in light petroleum in the presence of silica gel as a desiccant.

Because of the low solubility of DMS and its strong affinity to the paper, light petroleum and isopropyl ether were used as developing agents without being saturated with the stationary phase. With moist ethyl ether, $2 \% \mathrm{v} / \mathrm{v}$ of DMS was added if the chromatograms were to be developed for several hours with this solvent. The resulting impoverishment of the stationary phase in the upper parts of the paper will of course affect the migration of the spots but this occurs only to a small extent. When $\alpha$ - and $\beta$-glucose pentaacetates were run on chromatograms in DMS - light petroleum the average $R_{\mathrm{GA}}$ values 
Table 2. $R_{F}$ and $R_{G A}$ values of fully acetylated polyols. Stationary phase: DMS.

\begin{tabular}{ccc} 
Mobile phase: & $\begin{array}{c}\text { Light petroleum } \\
R_{\mathrm{GA}} \text { values }\end{array}$ & $\begin{array}{c}I_{\text {sopropyl ether }} \\
R_{F} \text { values }\end{array}$ \\
\hline Parent compound: & & \\
Glycerol & 5.6 & 0.43 \\
Erythritol & 4.2 & 0.42 \\
Adonitol & 3.0 & 0.39 \\
D-Arabitol & 3.1 & 0.37 \\
Xylitol & 1.1 & 0.23 \\
Dulcitol & 2.6 & 0.37 \\
L-Iditol & 0.44 & 0.16 \\
D-Mannitol & 3.7 & 0.42 \\
D-Sorbitol & 1.2 & 0.26 \\
Volemitol & 2.0 & 0.35 \\
D-Inositol & & \\
myo-Inositol & 1.3 & 0.31 \\
Laminitol & 0.19 & 0.12 \\
Mytilitol & 0.75 & 0.26 \\
Pinitol & 0.47 & 0.19 \\
& 1.6 &
\end{tabular}

(cf. Table 1 , note $a$ ) for the $\beta$-acetate were $0.43,0.47$ and 0.49 when the $\alpha$-acetate had travelled 3, 5 and $11 \mathrm{~cm}$, respectively. Using the method described above the reproducibility was tolerably good. However, when chromatograms were run for several hours it was often found that the distance travelled by a compound might vary considerably across the paper even if the solvent front not had been perceptibly oblique before leaving the paper. This error can be detected and corrected for by adding a reference compound at short intervals along the starting line. If this compound has a slightly higher $R_{F}$ value than the components of the samples it may be spotted on the paper at the same points as the samples to keep down the size of paper needed. The resolution is checked by running two reference compounds, the relative movements of which should be constant. Tables 2 and 3 list the $R_{F}$ or $R_{\mathrm{GA}}$ values of a number of acetylated sugar derivatives run in light petroleum or isopropyl ether on DMS treated paper.

As is apparent especially from the behaviour of the sugar alcohol acetates the mobilities of isomeric compounds show differences which could not be obtained previously in the paper chromatography of carbohydrates while the influence of the molecular weight is of secondary importance. This makes the new method a useful complement to the standard methods for paper chromatography in aqueous solvents where the opposite usually applies. The pyranosidic acetates and acetylated glycosides investigated should all have a $\mathrm{C} 1$ conformation, and it is interesting to note that in all cases the derivatives carrying an axial substituent on carbon atom 1 have much higher $R_{\mathrm{GA}}$ values than their respective anomers. This effect is more pronounced for the anomeric glycoside acetates of galactose than for the corresponding pentaacetyl deriva- 
Table 3. $R_{F}$ and $R_{\mathrm{GA}}$ values of fully acetylated monosaccharides and glycosides. Stationary phase: DMS

\begin{tabular}{|c|c|c|c|c|}
\hline \multirow{2}{*}{$\begin{array}{c}\text { Mobile phase: } \\
\text { Anomer: }\end{array}$} & \multicolumn{2}{|c|}{$\begin{array}{c}\text { Light petroleum } \\
R_{\mathrm{GA}} \text { values }\end{array}$} & \multicolumn{2}{|c|}{$\begin{array}{c}I s o \text { propyl other } \\
R_{F} \text { values }\end{array}$} \\
\hline & $\alpha$ & $\beta$ & $\alpha$ & $\beta$ \\
\hline $\begin{array}{l}\text { Parent compound: } \\
\text { D-Glucopyranose } \\
\text { D-Galactopyranose } \\
\text { D-Mannopyranose } \\
\text { L-Arabinopyranose } \\
\text { D-Xylopyranose } \\
\text { D-Fructopyranose }\end{array}$ & $\begin{array}{c}\left(R_{F}=0.024\right) \\
1.2 \\
0.69 \\
0.63 \\
2.2\end{array}$ & $\begin{array}{l}0.48 \\
0.33 \\
0.25 \\
1.6 \\
1.2 \\
0.40\end{array}$ & $\begin{array}{l}0.25 \\
0.30 \\
0.23 \\
0.17 \\
0.33\end{array}$ & $\begin{array}{l}0.15 \\
0.14 \\
0.12 \\
0.31 \\
0.23 \\
0.15\end{array}$ \\
\hline $\begin{array}{l}\text { Methyl D-glucopyranoside } \\
\text { Ethyl D-glucopyranoside } \\
\text { Methyl D-galactopyranoside } \\
\text { Ethyl D-galactopyranoside } \\
\text { Ethyl D-galactofuranoside } \\
\text { o-Cresyl D-galactopyranoside } \\
\text { 1-D-Glycerol D-galactopyranoside } \\
\text { 1-L-Glycerol D-galactopyranoside }\end{array}$ & $\begin{array}{l}2.3 \\
3.0 \\
6.4 \\
\\
5.9 \\
0.45 \\
0.45\end{array}$ & $\begin{array}{l}1.8 \\
0.6 \\
1.3 \\
4.3 \\
0.7 \\
0.08 \\
0.07\end{array}$ & $\begin{array}{l}0.29 \\
0.35 \\
0.49 \\
\\
0.52 \\
0.20 \\
0.20\end{array}$ & $\begin{array}{l}0.28 \\
0.18 \\
0.26 \\
0.41 \\
0.23 \\
0.08 \\
0.06\end{array}$ \\
\hline
\end{tabular}

tives and the difference also seems to increase with the bulk of the aglycon group. Although axial substitution in the 2- or 4-position usually seems to reduce the rate of migration, some $\alpha$-D-galactopyranose derivatives investigated do not obey this rule and so far the experimental material is too small for definite conclusions to be drawn.

Disadvantages of the method are that in some cases the procedures for detection of the spots are rather complicated and sometimes insensitive and also that the samples may have to be acetylated before chromatography. However, the difficulty of detecting a compound is often compensated by the fairly large quantities of material which can be run as a single spot. The acetylation is easily carried out on a micro scale and the low viscosity of the mobile solvents in combination with the high power of resolution usually allows the development to be completed within a few hours.

An important case where the standard method fails is in the separation of arabitol and adonitol as their acetates, since the relative rates of the spots are $1: 0.96$, respectively, in the system DMS - light petroleum. Substitution of FA or even better, a mixture of FA and DMF for the DMS gave complete resolution of the acetates, the relative rates of the components now being $1: 0.86-0.88$.

Treatment of sugar alcohols with formic acid and phosphorous pentoxide gives the corresponding fully formylated esters in fair yield ${ }^{10}$, and these may be run on paper chromatograms using DMS and ethyl or isopropyl ether as solvents. The mobilities are rather low, adonitol pentaformate travels about $1 \mathrm{~cm} / \mathrm{h}$ in isopropyl ether, and the relative rates for the adonitol, arabitol, xylitol and sorbitol derivatives were found to be $1.00: 0.74: 0.40$ :

Acta Chem. Scand. 12 (1958) No. 4 
Table 4. $R_{F}$ and $R_{\mathrm{GA}}$ values of 1,4-di-O-acetyl-2,3,6-tri- $O$-methyl derivatives of D-glucose and D-mannose. Stationary phase: DMS.

\begin{tabular}{lcc}
\hline Mobile phase: & $\begin{array}{c}\text { Light petroleum } \\
R_{\mathrm{GA}} \text { values }\end{array}$ & $\begin{array}{c}\text { Isopropyl ether } \\
R_{F} \text { values }\end{array}$ \\
\hline Parent compound: & & \\
$\alpha$-D-Glucose & 6.1 & 0.45 \\
$\beta$-D-Glucose & 4.9 & 0.42 \\
$\alpha-\mathrm{D}-$ Mannose & 1.8 & 0.25 \\
$\beta$-D-Mannose & 0.57 & 0.12
\end{tabular}

0.28 in the same solvent. Unfortunately the formates are not easily prepared on a micro scale, they are not very stable and some of them, e.g. those of mannitol, dulcitol and erythritol are unsuitable for paper chromatography because of their very low solubility.

Detailed experiments on the separation of methylated sugars in these solvents have not been carried out, but there are indications that DMS and ethyl or isopropyl ether may be used for chromatography of hexose tetramethyl ethers. Dr. I. Croon, Svenska Träforskningsinstitutet, has found that 2,3,6-tri$O$-methyl $\mathrm{D}$-glucose and 2,3,6-tri- $O$-methyl $\mathrm{D}$-mannose can be separated on DMS treated paper by developing with an ethyl ether - DMS (19:1) mixture. This separation is not easily accomplished with the usual aqueous solvent systems. However, while the mannose derivative gave mainly one spot (rate: $5.4 \mathrm{~cm} / 6 \mathrm{~h}$ ) the glucose derivative gave two (rates: 6.2 and 8.0 $\mathrm{cm} / 6 \mathrm{~h}$ ), the relative intensities of which changed on storing the sample solution. Probably the mutarotation in this case was slow enough to allow the separation of the $\alpha$ - and $\beta$-anomers. A similar phenomenon was observed for 2,3,4,6-tetra-O-acetyl D-glucose (see Table 1 ). To avoid this complication and to obtain derivatives with higher mobilities, milligram samples of the trimethyl ethers were acetylated with pyridine, sulphuric acid or sodium acetate as catalyst. The resulting products were subjected to paper chromatography using DMS as the stationary phase (Table 4). The spots were detected by spraying with anisidine hydrochloride (see Experimental part). Since the acetyl derivatives are somewhat volatile the chromatograms were not dried before spraying with hexamethylene diamine solution. The structures were assigned on the assumption that the catalysts directed the formation of $\alpha$-and $\beta$-acetates in the usual way. In the presence of sulphuric acid or pyridine the mannose derivative yielded the $\alpha$-acetate almost exclusively and even with sodium acetate as catalyst the same anomer was still the major product. In the case of the glucose trimethyl ether the $\beta$-acetate was the more favoured anomer.

The very different $R_{\mathrm{GA}}$ values found for the glucose and mannose derivatives (Table 4) shows that paper chromatography of acetylated sugar methyl ethers may be a useful method for both identification and isolation.

Separation on a preparative scale of closely related compounds may occasionally be effected by a simple counter-current extraction technique as is illustrated by the preparation of $o$-cresyl $\alpha$-and $\beta$-D-galactopyranoside tetraacetate. From the mixture of acetylated galactosides which is formed on heating 
penta- $O$-acetyl $\beta$-D-galactopyranose with $o$-cresol in the presence of zinc chloride Helferich et al. ${ }^{11}$ isolated the pyranosidic $\alpha$-and $\beta$-derivatives by fractional crystallisation, but this separation was now found to be very unpredictable. As calculated from the $R_{F}$ values of the $\alpha$-and $\beta$-compounds using DMS and moist ethyl ether as solvents $\left(R_{F} c a .0 .9\right.$ and 0.8 , resp.) the corresponding partition coefficients $\left(C_{\text {ether }} / C_{\mathrm{DMS}}\right)$ were in the approximate ratio $2.3: 1$. Separation of the mixture by counter-current extraction was therefore attempted using ethyl ether and DMS containing $5 \% \mathrm{v} / \mathrm{v}$ of water as solvents. The process was followed on paper chromatograms and by a suitable withdrawal of enriched fractions only six separating funnels were required. The pure components could finally be isolated in a good yield.

\section{EXPERIMENTAL}

Paper. Whatman No. 1 filter paper was used throughout.

Solvents. All solvents were of reagent grade. Formamide (FA), dimethyl formamide (DMF) and dimethyl sulphoxide (DMS)* were distilled under reduced pressure; other solvents were used without purification. The light petroleum had b.p. 60-71 ${ }^{\circ}$ (Skellysolve B).

Impregnation with the stationary phase. In the standard procedure for impregnation with DMS the chromatogram papers were passed at an even rate through a $25 \% \mathrm{v} / \mathrm{v}$ solution of DMS in toluene, uniformly blotted between layers of filter paper and then dried at $60^{\circ}$ for $1 \mathrm{~min}$ to remove the toluene. This process was repeated once and after the second drying the papers were immediately placed between glass plates leaving only the starting line and paper above it uncovered. After application of the spots the papers were transferred to the chromatogram tank in such a manner that absorption of atmospheric moisture was avoided.

When the reproducibility is of secondary importance the above procedure may be simplified, e. $g$. by blowing off the toluene from the papers with hot air. If the papers are to be handled without protection from moisture it is advisable to impregnate with a more dilute DMS solution, e.g. $20 \%$.

In the preliminary experiments on acetylated compounds (Table 1) the following impregnating solutions were used: $20 \% \mathrm{v} / \mathrm{v}$ FA in ethyl acetate; $20 \% \mathrm{v} / \mathrm{v}$ DMF in benzene; $20 \% \mathrm{v} / \mathrm{v}$ DMS in benzene. Each paper was dipped in the impregnating solution, blotted and dried at $60^{\circ}$ for 30 sec. After a second impregnation in the same manner the papers were dried at $60^{\circ}$ for $1 \mathrm{~min}$ and then covered with glass slides while the samples were applied.

For separation of the pentaacetates of arabitol and adonitol using FA as the stationary phase the papers were impregnated twice with a $20 \% \mathrm{v} / \mathrm{v}$ solution of FA in ethyl acetate analogous to the DMS treatment described above. To obtain a mixed FA-DMF phase the second impregnation was made with a $20 \% \mathrm{v} / \mathrm{v}$ solution of DMF in benzene.

Application of samples. The samples were usually applied as $2 \%$ solutions in ethyl ether or chloroform but provided that no crystallisation occurred upon drying the concentration could be raised to 5 or even $10 \%$.

Development. All chromatograms were run by the descending technique. In view of the fire hazard and for general convenience, small glass tanks were used for development with benzene, isopropyl ether or ethyl ether, the papers being ca $23 \mathrm{~cm}$ long with the starting line $5 \mathrm{~cm}$ from the upper end. Where not otherwise stated, separations in light petroleum were made on papers ca $46 \mathrm{~cm}$ long with the starting line $7 \mathrm{~cm}$ from the upper end and with silica gel present as a desiccant in the chromatogram tank. The solvent reached the lower end in 25-35 min for the short papers and 60-90 min for the long ones.

Detection of spots. a) Silver nitrate - sodium hydroxide spray ${ }^{8}$. After drying for 5-10 $\min$ at $120^{\circ}$ the chromatograms were sprayed with aqueous silver nitrate (saturated aqueous solution - water $1: 100 \mathrm{v} / \mathrm{v}$ ) and dried in a current of hot air, excessive heating

* Purchased from Nitroglycerin AB, Gyttorp, Sweden.

Acta Chem. Scand. 12 (1958) No. 4 
being avoided. (While the paper was still wet some compounds appeared as greasy spots.) The chromatograms were then sprayed on both sides with an alcoholic sodium hydroxide solution prepared from 1 part of an aqueous solution $(40 \mathrm{~g} \mathrm{NaOH}$ in $100 \mathrm{ml}$ water) and 9 parts of ethanol. Some compounds, e.g. acetates of reducing sugars reacted quickly while others like acetylated alkyl glycosides gave spots very slowly. In the latter case it was found favourable to keep the wet chromatograms after spraying with alkali between glass slides to prevent evaporation of ethanol. As soon as possible and before the background had become too dark the papers were washed successively with dilute ammonia and water and finally treated with aqueous hydrogen sulphide. (Prolonged treatment with ammonia or hydrogen sulphide has a bleaching action.) This method could not be used for chromatograms impregnated with FA.

b) Hexamethylene diamine. Commercial $70 \%$ aqueous hexamethylenediamine solu. tion was distilled under reduced pressure and the distillate was diluted with 4 parts of ethanol. When chromatograms were sprayed with this reagent and then heated at $120^{\circ}$ over a period of 5-10 min the acetates of reducing sugars showed up as faint yellowish spots which were strongly fluorescent in ultraviolet light. Usually the chromatograms were dried for 5-10 $\mathrm{min}$ at $120^{\circ}$ before spraying, but in the case of DMS this was found to be unnecessary.

c) p-Anisidine hydrochloride. After treatment as in b) to remove the acetate groups the chromatograms were sprayed with a solution of $p$-anisidine $(2.3 \mathrm{~g})$ in a mixture of concentrated hydrochloric acid $(5 \mathrm{ml})$, ethanol $(30 \mathrm{ml})$ and butanol $(60 \mathrm{ml})$. After a fow minutes heating at $120^{\circ}$ the chromatograms were inspected by visible and ultraviolet light. The acetates of the 2,3,6-trimethyl ethers of glucose and mannose showed up as bright red-violet spots and glucose pentaacetates as light brown spots. The chromatograms were then rinsed with water.

d) Sodium periodate ${ }^{\circ}$. After treatment as in b) the chromatograms were dipped in $90 \%$ aqueous acetone containing $10 \%$ phthalic acid, dried and then sprayed with $0.2 \%$ aqueous sodium metaperiodate. After ca $5 \mathrm{~min}$ they were sprayed with a reagent prepared by mixing equal parts of $5 \%$ aqueous manganese sulphate and $5 \%$ 4,4-methylenebis ( $N, N$-dimethyl aniline) in $2 \mathrm{~N}$ aqueous formic acid. On drying in a current of hot air, white spots appeared against a blue-green background. The spots were unstable to prolonged heating and disappeared after a few days at room temperature. Insufficient acidification with phthalic acid was found to give rise to an uneven background, the colour partly appearing before the chromatograms were dried. Acetates of sugar alcohols and their glycosides were easily detected but in the case of acetylated alkyl glycosides and cyclitols the sensitivity was less satisfactory.

Acetylation of O-methyl sugars. 8) Acetic anhydride - pyridine. A sample (1-2 mg) of the sugar was dissolved in acetic anhydride $(30 \mu 1)$ and pyridine $(30 \mu 1)$ in a capillary tube and the solution was heated to $100-105^{\circ}$ for $5 \mathrm{~min}$. After decomposition of unreacted acetic anhydride with a few drops of water the product was extracted with chloroform $(0.2 \mathrm{ml})$ and the extract successively washed with dilute sulphuric acid, aqueous sodium bicarbonate and water and finally dried with magnesium sulphate. When applied to paper chromatograms 5-10 4 l of this solution were put on the same spot.

b) Acetic anhydride - sulphuric acid. The sample (1-2 mg) dissolved in acetic anhydride $(40 \mu \mathrm{l})$ containing $1 \% \mathrm{v} / \mathrm{v}$ of sulphuric acid was heated to $100^{\circ}$ for $1 \mathrm{~min}$. The solution especially in the case of 2,3,6-tri-O-methyl D-mannose turned very dark. After cooling and adding 2 drops of pyridine the mixture was worked up as under a).

c) Acetic anhydride - sodium acetate. A mixture of the sample (1-2 mg), acetic anhydride $(40 \mu \mathrm{l})$ and a few crystals of anhydrous sodium acetate was heated to $110^{\circ}$ for $1 \mathrm{~h}$. Pyridine (2 drops) was added after cooling and the product isolated as above.

Separation of anomeric o-cresyl D-galactopyranoside tetraacetates by counter-current extraction. $\beta$-pentaacetyl $\mathrm{D}$-galactose $(93 \mathrm{~g})$ was added with stirring to a mixture of 0 -cresol $(85 \mathrm{~g})$ and anhydrous zinc chloride $(5 \mathrm{~g}) \mathrm{kept}$ at $120^{\circ}$. The temperature was then raised to $135-140^{\circ}$ for 40 mins. After cooling the reaction mixture was dissolved in benzene and the solution was washed with $2 \mathrm{~N}$ aqueous sodium hydroxide and water, dried with sodium sulphate and concentrated to dryness. The residue, dissolved in dilute ethanol gave a crude crystalline mixture of the anomeric o-cresyl D-galactopyranoside tetraacetates.

The above acetate mixture was added to No. 1 of a series of six separating funnels each of which contained ethyl ether $(300 \mathrm{ml})$ saturated with DMS containing $5 \% \mathrm{v} / \mathrm{v}$ 
of water. Successive $75 \mathrm{ml}$ portions of DMS, containing $5 \%$ of water and saturated with ether, were used for the extraction. At the stage at which DMS portion No. 1 had reached funnel No. 6, funnel No. 1 contained DMS portion No. 6, etc. The separation was followed by chromatograms of the ether phase. The chromatograms were run for about 5 mins in DMS-isopropyl ether. When ether phase No. 1 was sufficiently free from the $\beta$-anomer (after seven extractions) it was withdrawn and the funnel after being refilled with fresh ether phase placed as No. 7 in the series of funnels and the extraction continued. When possible the number of funnels was reduced one by one. When only one remained the contents of this were discarded. Altogether 18 portions of ether and 35 of DMS were required. The combined ether solutions were freed from DMS by washing with water, concentrated and the residue was recrystallised yielding crude o-cresyl $a-\mathbf{D}$ galactopyranoside tetraacetate $(43 \mathrm{~g})$, m. p.* $80-90^{\circ},[\alpha]_{\mathrm{D}}+160^{\circ}$ (chloroform, $c=2$ ). By paper chromatography it was shown to contain traces of the $\beta$-anomer. The pure compound with m. p. $90-92^{\circ}$ and $[\alpha]_{\mathrm{D}}+176^{\circ}$ was obtained by treating the crude product with $2 \mathrm{~N}$ aqueous sodium hydroxide $(1400 \mathrm{ml})$ at $100^{\circ}$ for $16 \mathrm{~h}$ and reacetylating the hydrolysed material (yield $31 \mathrm{~g}$ ).

The combined DMS solutions were diluted with water and extracted with ether. The ether solution was concentrated and the residue recrystallised giving pure 0 -cresyl $\beta$-D-galactopyranoside tetraacetate $(22 \mathrm{~g}), \mathrm{m} . \mathrm{p} .114-116^{\circ},[\alpha]_{\mathrm{D}} \pm 0$.

Acknowledgement. The author is indebted to Statens Naturvetenskapliga Forskningsrdd for financial support, to Ing. Gurli Hammarberg for her skilful assistance and to Dr. B. Thomas for checking the English.

* All melting points are corrected.

\section{REFERENCES}

1. Baddiley, J., Buchanan, J. G. and Carss, B. J. Chem. Soc. 19574138.

2. Zaffaroni, A., Burton, R. B. and Keutmann, E. H. Science 111 (1950) 6.

3. Schindler, O. and Reichstein, T. Helv. Chim. Acta 34 (1951) 108.

4. Jörgensen, C. and Kofod, H. Acta Chem. Scand. 8 (1954) 941.

5. Kaiser, F. Chem. Ber. 88 (1955) 556.

6. Mitchell, L. C. J. Assoc. Offic. Agr. Chemists 37 (1954) 216; from Chem. Abstr. 48 (1954) 14088 e.

7. Micheel, F. and Schweppe, H. Mikrochim. Acta 195453.

8. Trevelyan, W. E., Procter, D. P. and Harrison, J. S. Nature 166 (1950) 444.

9. Yoda, A. J. Chem. Soc. Japan Pure Chem. Sect. 73 (1952) 18; from Chem. Abstr. 47 (1953) 3185 c.

10. Wickberg, B. Acta Chem. Scand. 12 (1958) 624.

11. Helferich, B., Scheiber, H. E., Streөck, R. and Vorsatz, F. Ann. 518 (1935) 211.

Received February 14, 1958. 